Kompass

Autoimmun

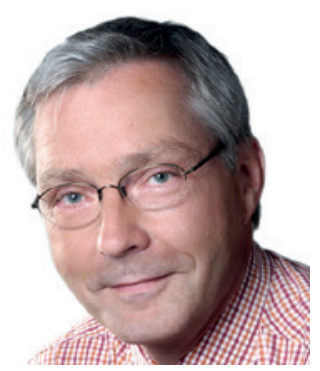

\title{
Immuncheckpoint-Inhibitoren vermitteln ein großes Spektrum von unerwünschten immunvermittelten Reaktion an der Haut
}

\author{
Michael Sticherling \\ Universitätsklinikum Erlangen, Hautklinik, Erlangen, Deutschland
}

\begin{abstract}
Wongvibulsin S, Pahalyants V, Kalinich M, et al.: Epidemiology and risk factors for the development of cutaneous toxicities in patients treated with immune-checkpoint inhibitors: a United States population-level analysis. J Am Acad Dermatol 2021, in press. DOI: 10.1016/j.jaad.2021.03.094.
\end{abstract}

\author{
Keywords \\ Cutaneous · Dermatologic · Drug reactions · Immune- \\ checkpoint inhibitors $\cdot$ Immune-related adverse events. \\ Immunotherapy
}

\begin{abstract}
Background: A variety of dermatoses have been reported in the growing number of patients treated with immune-checkpoint inhibitors (ICls), but the current understanding of cutaneous immune-related adverse events (irAEs) is limited.

Objective: To determine the cumulative incidence, distribution, and risk factors of cutaneous irAEs after $\mathrm{ICl}$ initiation.

Methods: This was a retrospective cohort study of patients in a national insurance claims database including cancer patients treated with $\mathrm{ICls}$ and matched controls.

Results: The study included $8637 \mathrm{ICl}$ patients and 8637 matched controls. The overall incidence of cutaneous irAEs was $25.1 \%$, with
\end{abstract}

a median onset time of 113 days. The $\mathrm{ICl}$ group had a significantly higher incidence of pruritus, mucositis, erythroderma, maculopapular eruption, vitiligo, lichen planus, bullous pemphigoid, Grover disease, rash, other nonspecific eruptions, and drug eruption or other nonspecific drug reaction. Patients with melanoma and renal cell carcinoma and those receiving combination therapy were at a higher risk of cutaneous irAEs.

Limitations: Retrospective design without access to patient chart data.

Conclusions: This study identifies cutaneous irAEs in a realworld clinical setting and highlights patient groups that are particularly at risk. The results can aid dermatologists at the bedside in the diagnosis of cutaneous irAEs and in formulating management recommendations to referring oncologists regarding the continuation of $\mathrm{ICl}$ therapy.

C 2020 American Academy of Dermatology, Inc.

Abstract aus Molina GE, Reynolds KL, Chen ST: Diagnostic and therapeutic differences between immune checkpoint inhibitor-induced and idiopathic bullous pemphigoid: a cross-sectional study. Br J Dermatol 2020;183:1126-1128.

\begin{abstract}
Bullous pemphigoid (BP) is a serious, rare complication from immune checkpoint inhibitors (ICls). While most dermatologic immune-related adverse events (irAEs) present early and are mild, ICl-induced BP (ICl-BP) manifests months into therapy and can prompt $\mathrm{ICl}$ discontinuation and severe immunosuppression. Fol-
\end{abstract}

lowing irAE management guidelines, providers rely on systemic corticosteroids - which, at high doses, may confer poorer oncologig outcomes for $\mathrm{ICl}$ patients. Our study aims to identify diagnostic and therapeutic differences between ICI-BP and idiopathic BP (iBP) to assist providers in prompt recognition and management of this skin toxicity. 


\section{Transfer in die Praxis}

\section{Hintergrund}

Die Immunabwehr ist unter regulierten Bedingungen in der Lage, Autoreaktivität effektiv zu verhindern (bestehende Sensibilisierungen zu unterdrücken) oder gar zu eliminieren (sensibilisierte immunkompetente Zellen zu zerstören). Tumorzellen können genau diese Schutzmechanismen für ihr Überleben ausnutzen und damit der Immunabwehr entgehen. Eine therapeutische Beeinflussung dieser Mechanismen durch die modernen Immuncheckpoint-Inhibitoren (ICI) ist einerseits in der Lage, eine ganze Reihe von malignen Tumoren sehr effektiv zu bekämpfen, kann aber andererseits gleichzeitig zur Induktion von klinisch manifesten Entzündungen des Darms, der Leber, der Hirnanhangsdrüse, der Nieren und Lunge, aber auch der Haut führen.

\section{Ergebnisse der Studien}

Im Folgenden sollen 2 jüngste Publikationen referiert werden, die entzündliche und autoimmune Erkrankungen der Haut unter $\mathrm{ICl}$ darstellen.

Die Häufigkeit und Risikofaktoren für die Entwicklung immunologisch vermittelter kutaner Nebenwirkungen als Erkrankungsgruppe bei Patient*innen unter $\mathrm{ICl}$ haben Wongvibulsin et al. in einer Kohortenstudie aus den USA untersucht. Retrospektiv wurden dafür Daten einer nationalen Krankenversicherung erfasst und 8600 Patient*innen unter ICI-Therapie sowie 8600 angepasste Kontrollen eingeschlossen. Die Gesamtinzidenz von immunologisch vermittelten kutanen Nebenwirkungen betrug 25\% mit einer Manifestation im Mittel 113 Tage nach der Applikation. Die betreffenden Patient*innen hatten eine höhere Wahrscheinlichkeit von Juckreiz, Mukositis, Erythrodermie, makulopapulösem Exanthem, Vitiligo, Lichen planus und bulllösem Pemphigoid sowie Medikamenten-assoziiertem Exanthem und anderen unspezifischen kutanen Manifestationen. Patient*innen mit Melanom und Nierenzellkarzinom sowie solche, die eine Kombinationstherapie erhielten, hatten das höchste Risiko für immunologisch vermittelte kutane Unverträglichkeitsreaktionen.

Molina et al. berichten in einem «Letter to the Editor» des BRITISH JOURNAL OF DERMATOLOGY von einer spezifischen Autoimmunerkrankung der Haut, dem bullösen Pemphigoid (BP). Sie präsentieren Ergebnisse einer multizentrischen Datenerfassung von $\mathrm{ICl}$-induziertem BP (ICl-BP) und im Verhältnis 1:2 von idiopathischem BP (BPP). In der Zeit von 2015 bis 2018 konnten sie 15 Patient*innen erfassen, die alle PD-L1-Inhibitoren erhalten hatten (10 Pembrolizumab, 5 Nivolumab), 20\% davon Frauen mit einem mittleren Alter von 71,5 Jahren. Die genauen Tumorentitäten werden im Artikel nicht aufgeführt. BP-Symptome traten im Median nach 11 Zyklen und 266 Tage nach der ersten ICl-Applikation auf. Bei 87\% der Patient*innen führte die Hauterkrankung zu einer Verzögerung der ICl-Gabe oder zum vollständigen Abbruch der Therapie. Interessant war, dass die Patient*innen eine signifikant längere Phase von Juckreiz ohne Hautveränderungen sowie mit einer Odds Ratio (OR) von 0,17 deutlich weniger häufig pralle Blasen oder eine subepider- male Spaltbildung in der histologischen Untersuchung zeigten. IClBP-Patient*innen hatten deutlich häufiger eine periphere Eosinophilie $(\mathrm{OR}$ 13,1). Auch das Intervall zwischen ersten Symptomen wie Juckreiz oder Hautveränderungen bis zur BP-Diagnose war deutlich länger als bei Kontrollen (110 vs. 92 Tage). Die Therapie entsprach der bei iBP, wobei systemische Kortikosteroide deutlich häufiger (93\% vs. 50\% der Kontrollen) angewendet wurden, Tetrazykline hingegen in beiden Gruppen ähnlich häufig (67\% vs. 50\%). Patienten mit ICl-BP sprachen jedoch deutlich seltener an (0\% vs. 40\%). Insgesamt war damit bei ICI-BP die Zahl von Therapieansätzen ohne Erfolg deutlich höher als bei iBP (Median 2 vs. 0).

\section{Kommentar}

Die $\mathrm{ICl}$, die zentrale Stellen der immunologischen Tumorabwehr beeinflussen und zur Therapie unter anderem des metastasierenden Melanoms zugelassen sind, zeigen frappante klinische Effekte, daneben aber eine Reihe schwerer unerwünschter Wirkungen. Hier zeigt sich besonders eindrucksvoll, dass neben Fieber, Exanthemen, Ödemen und Juckreiz die zu beobachtenden Entzündungen des Darms, der Leber, der Hirnanhangsdrüse, der Nieren, der Lunge und auch der Haut möglicherweise autoimmunologisch oder autoreaktiv bedingt sind.

Die Erkrankungen der Pemphigoid-Gruppe zeichnen sich durch Antikörper gegen verschiedene Antigene der Basalmembranzone aus und zeigen eine klinisch ähnliche Symptomatik. Das BP ist dabei die häufigste autoimmunbullöse Dermatose mit einem Altersgipfel im 7. Lebensjahrzehnt. Klinisch finden sich pralle Blasen auf erythematösem Grund, in der Immunfluoreszenzuntersuchung eine bandförmige Ablagerung von Immunglobulin G (lgG) und Komplementfaktor C3. Das häufigste gemeinsame Zielantigen der Erkrankungen dieser Gruppe ist das BP-Antigen 180 kD (BP180) oder Kollagen XVII. Therapie der ersten Wahl bleiben zumindest bei ausgedehntem BP mit deutlichem Entzündungsdruck bis heute systemische Kortikosteroide in Kombination mit verschiedenen Adjuvantien, allen voran Azathioprin und Mycophenolatmofetil. Alternativ können Dapson oder Tetrazykline eingesetzt werden, bei milderen Krankheitsformen auch als Monotherapie. Die Therapie mit der höchsten Evidenz (doppelblinde Placebo-kontrollierte klinische Studie) ist jedoch die langfristige und gegebenenfalls großflächige Nutzung von Clobetasoldiproprionat-Creme. Bei umschriebenen Formen eines BP und geringem Blasenschub ist die topische Therapie nebenwirkungsarm und einer Systemtherapie vorzuziehen.

In der Vergangenheit sind zahlreiche Medikamente verdächtigt worden, ein BP auszulösen. Das Spektrum ist dabei breit sowie sehr heterogen und umfasst Diuretika, Penicillin, nichtsteroidale Antirheumatika (NSAID), Angiotensin-Converting-Enzyme (ACE)-Hemmer, Dipeptidylpeptidase-4-Inhibitoren und ICI. Vor einem immungenetischen Hintergrund führen diese Medikamente bei entsprechend prädisponierten Individuen zur Demaskierung von Autoantigenen oder durch Derivatisierung zur Generierung von 
Neoantigenen mit nachfolgenden immunologischen Prozessen. Bei ICl sind direkt Mechanismen der Immunregulation betroffen, die Autoimmunprozesse in Gang setzen und ein möglicherweise eigenständiges, vom klassischen Medikamenten-induzierten BP differentes Krankheitsbild induzieren. Der Exanthem-freie Pruritus ist viel stärker und eine nichtbullöse Manifestation deutlich häufiger, auch ist offensichtlich häufiger eine systemische immunsuppressive Therapie nötig. Die klinischen Konsequenzen sind daher individuell zu ziehen, indem insbesondere bei neu angesetzten Medikamenten und Erstmanifestation bzw. Exazerbation eines BP eine Pausierung zu überlegen ist.

\section{Fazit}

Die ICl, die zentrale Stellen der immunologischen Tumorabwehr beeinflussen und zur Therapie des metastasierenden Melanoms zugelassen sind, zeigen frappante klinische Effekte, daneben aber eine Reihe schwerer unerwünschter Wirkungen wie Kolitiden, Hy- pophysitis, aber auch das Auftreten von Autoimmunerkrankungen. Vorbestehende, klinisch minimal ausgeprägte oder inapparente Autoimmunerkrankungen werden exazerbiert oder vor dem Hintergrund einer immunologischen Prädisposition erstmalig klinisch manifest. Vor Einleitung einer ICl-Therapie sollten daher Autoimmunerkrankungen abgefragt und ausgeschlossen werden, wobei klare Empfehlungen zu Art und Umfang solcher Untersuchungen bisher nicht gemacht sind. Zusätzlich sollte bei Juckreiz frühzeitig an ein BP gedacht werden, zumal Juckreiz selbst durch die Tumortherapie induziert werden kann.

\section{Disclosure Statement}

Kein Interessenskonflikt.

Korrespondenz an:

Michael Sticherling, michael.sticherling @ uk-erlangen.de 